Supplementary Information for

\title{
A Synthetic Multi-Domain Peptide That Drives a Macropinocytosis-Like Mechanism for Cytosolic Transport of Exogenous Proteins into Plants
}

Takaaki Miyamoto ${ }^{1}$, Kiminori Toyooka $^{2}$, Jo-Ann Chuah ${ }^{1}$, Masaki Odahara ${ }^{1}$, Mieko Higchi-Takeuchi ${ }^{1}$, Yumi Goto $^{2}$, Yoko Motoda ${ }^{1,3}$, Takanori Kigawa ${ }^{3}$, Yutaka Kodama ${ }^{4} \&$ Keiji Numata ${ }^{1,5 *}$

${ }^{1}$ Biomacromolecules Research Team, RIKEN Center for Sustainable Resource Science, Saitama 351-0198, Japan.

2 Technology Platform Division, Mass Spectrometry and Microscopy Unit, RIKEN Center for Sustainable Resource Science, Yokohama, 230-0045, Japan

${ }^{3}$ Laboratory for Cellular Structural Biology, RIKEN Center for Biosystems Dynamics Research, Yokohama 230-0045, Japan.

${ }^{4}$ Center for Bioscience Research and Education, Utsunomiya University, Tochigi 321-8505, Japan.

${ }^{5}$ Department of Material Chemistry, Graduate School of Engineering, Kyoto University, Kyoto-Daigaku-Katsura, Nishikyoku, Kyoto 615-8510, Japan.

*Corresponding author: Keiji Numata (E-mail: numata.keiji.3n@kyoto-u.ac.jp)

\section{Contents}

Supplementary Methods

p. S2

Supplementary Figures

Figure S1 Viability of BY-2 cells after prolonged treatment with dTat-Sar-EED4 for up p. S3 to $48 \mathrm{~h}$.

Figure S2 Confocal images showing negligible Citrine uptake in BY-2 cells treated p. S4 with low concentrations of dTat-Sar-EED4 or dTat-Sar-EED5.

Figure S3 Confocal images of BY-2 cells, $A$. thaliana cotyledons and $O$. sativa calli $\quad$ p. S5 after GUS transduction with dTat-Sar-EED4 under different conditions.

Figure S4 Confocal images of BY-2 cells, A. thaliana cotyledons and $O$. sativa calli $\quad$ p. S6 after GUS transduction with dTat-Sar-EED5 under different conditions.

Figure S5 Confocal images of BY-2 cells, A. thaliana cotyledons and $O$. sativa calli $\quad$ p. S7 after GUS transduction with or without various types of peptides.

Figure S6 Confocal images of A. thaliana root cells treated for 30 min with water or p. S8 DMSO and then stained for 30 min with FM4-64 $(10 \mu \mathrm{M})$.

$\begin{array}{lll}\text { Figure S7 GUS enzymatic activity in the presence of pharmacological inhibitors. } & \text { p. S9 }\end{array}$

Figure S8 Transmission electron microscopic (TEM) images showing the formation of p. S10 plasma membrane protrusions with unstructured objects in dTat-Sar-EED4treated $A$. thaliana roots.

Figure S9 TEM images of BY-2 cells treated without and with dTat-Sar-EED4. p. S11

\section{Supplementary Movies}


Movie S1 Citrine uptake by BY-2 cells in the presence of $90 \mu \mathrm{M}$ dTat-Sar-EED4.

Movie S2 Citrine uptake by BY-2 cells in the presence of $90 \mu \mathrm{M}$ dTat as a control.

\section{Supplementary Methods}

In vitro GUS enzymatic activity in the presence of pharmacological inhibitors. GUS ( $25 \mu \mathrm{g} / \mathrm{mL}$, Nacalai Tesque) was mixed with dye-BGlcU (20 $\mu \mathrm{M}$, Goryo Chemical) in the presence of dTat-Sar-EED4 $(90 \mu \mathrm{M})$ and pharmacological inhibitors such as ES9-17 (100 $\mu \mathrm{M}$, Carbosynth), wortmannin (40 $\mu \mathrm{M}$, Fujifilm) and cytochalasin D (40 $\mu \mathrm{M}$, Fujifilm) in half-strength Murashige and Skoog (1/2 MS) medium (100 $\mu \mathrm{L})$ containing $1 \%$ sucrose. Following incubation at $25{ }^{\circ} \mathrm{C}$ for $10 \mathrm{~min}$, the mixture was transferred onto a 96 -well microplate and fluorescence intensity was measured using a multimode microplate reader (Spectra MAX M3, Molecular Devices Corporation) with excitation and emission wavelengths of $485 \mathrm{~nm}$ and $535 \mathrm{~nm}$, respectively. The fluorescence intensity of each sample was normalized to that measured in the absence of inhibitors to obtain the relative GUS activity. 


\section{Supplementary Figures}

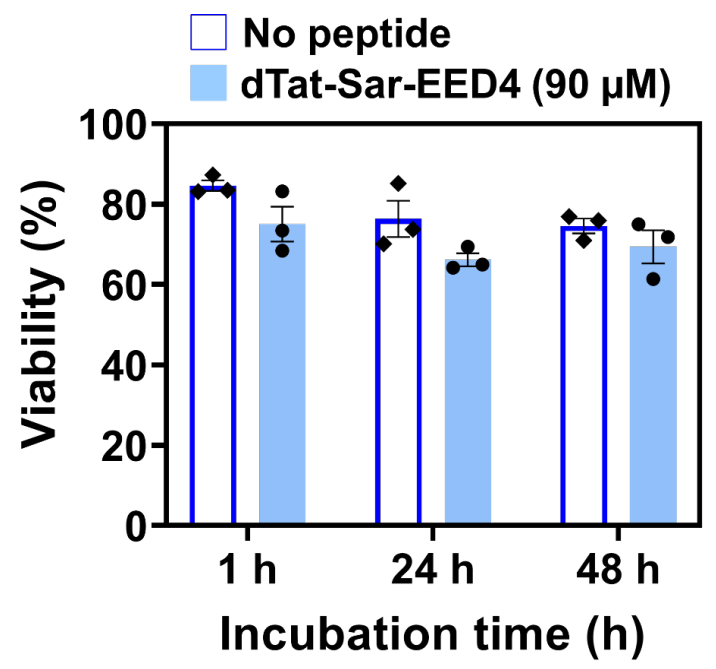

Figure S1. Viability of BY-2 cells after prolonged treatment with dTat-Sar-EED4 for up to $48 \mathrm{~h}$. Cell viability was determined by Evans blue assay. Data from three biological replicates are represented as the mean \pm standard error values. 


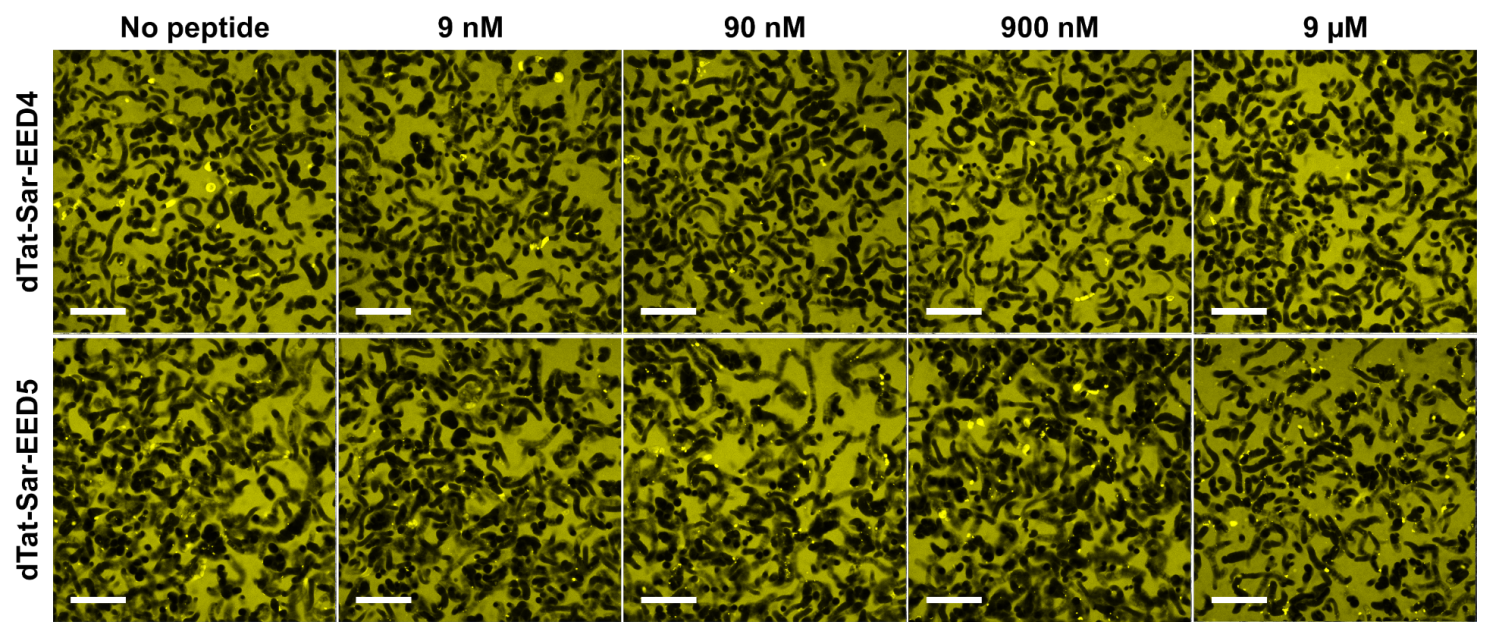

Figure S2. Confocal images showing negligible Citrine uptake in BY-2 cells treated with low concentrations of dTat-Sar-EED4 or dTat-Sar-EED5. Scale bars, $200 \mu \mathrm{m}$. 


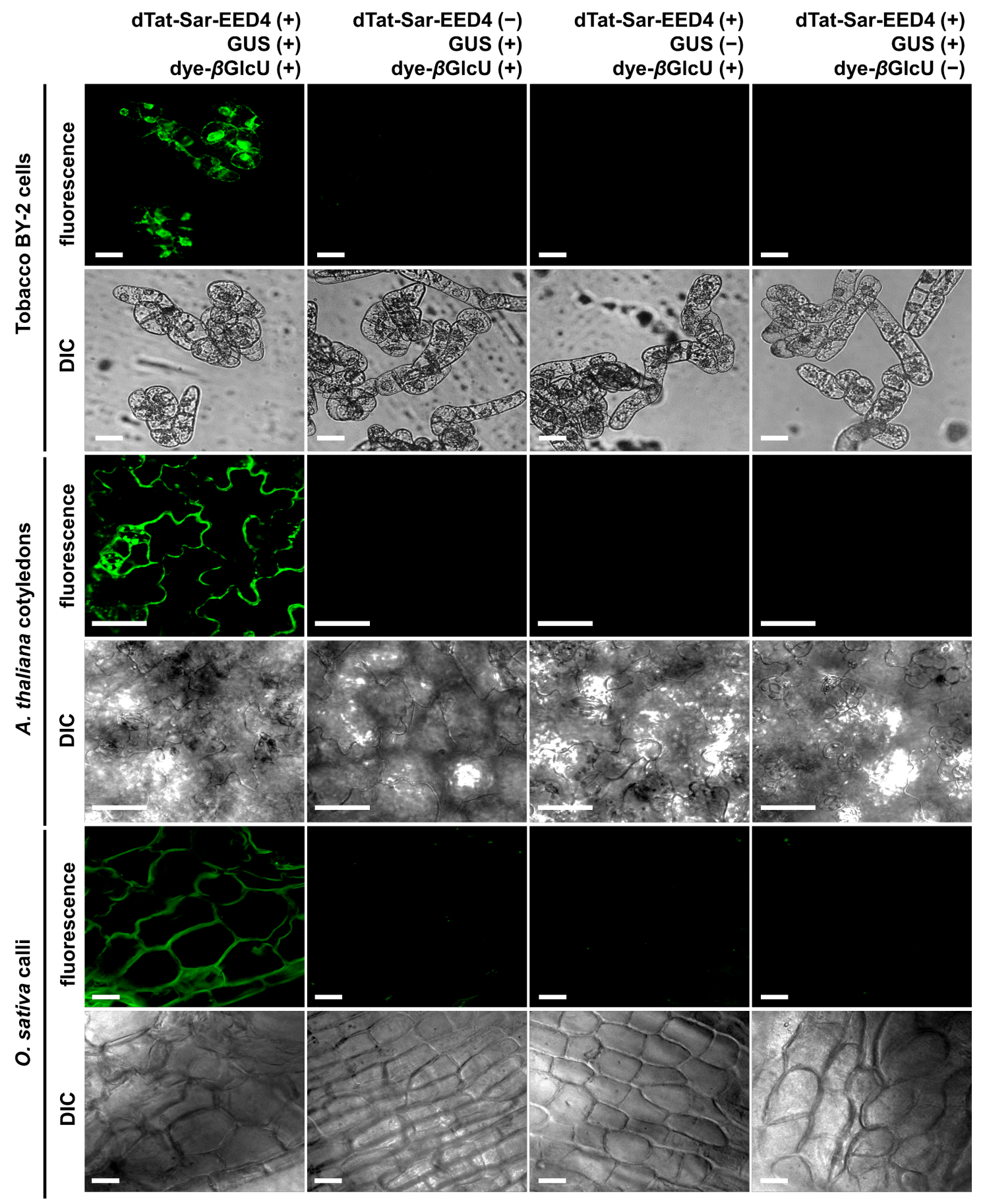

Figure S3. Confocal images of BY-2 cells, $A$. thaliana cotyledons and $O$. sativa calli after GUS transduction with dTat-Sar-EED4 under different conditions. Samples treated with GUS and dTat-Sar-EED4 ( $90 \mu \mathrm{M})$ for $30 \mathrm{~min}$, and then with dye- $\beta \mathrm{GlcU}(10 \mu \mathrm{M})$ for $15 \mathrm{~min}$, exhibited green fluorescence of the dye produced by GUS-catalyzed hydrolysis in the cytosol. Conversely, no green fluorescence was observed in the absence of dTat-Sar-EED4, GUS or dye- $\beta$ GlcU. Excess GUS and dTat-Sar-EED4 were washed out before dye- $\beta$ GlcU treatment, while excess dye- $\beta \mathrm{GlcU}$ was removed prior to confocal microscopic observation. DIC, differential interference contrast. Scale bars, $40 \mu \mathrm{m}$. 


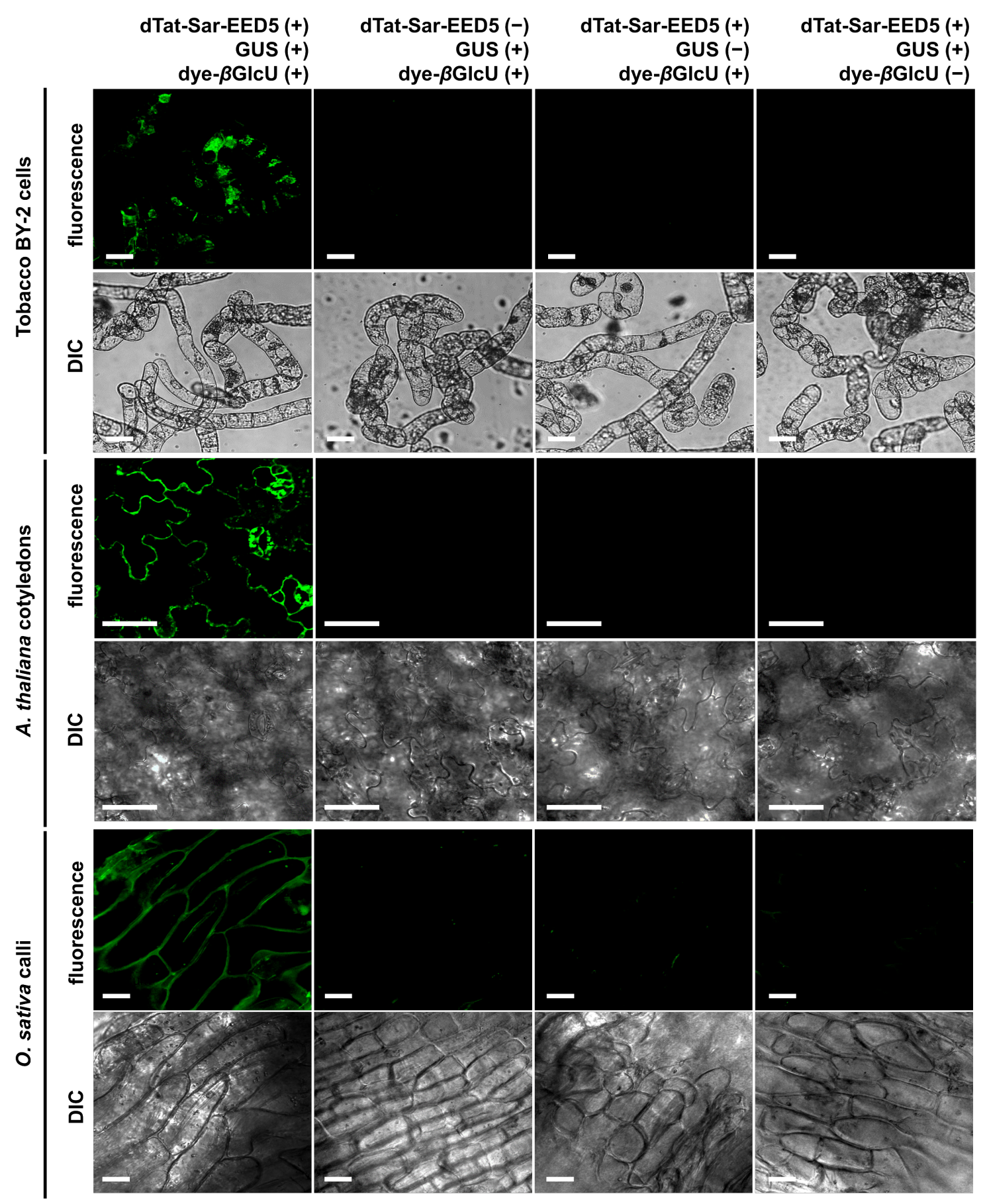

Figure S4. Confocal images of BY-2 cells, $A$. thaliana cotyledons and $O$. sativa calli after GUS transduction with dTat-Sar-EED5 under different conditions. Samples treated with GUS and dTat-Sar-EED5 ( $90 \mu \mathrm{M})$ for $30 \mathrm{~min}$, and then with dye- $\beta \mathrm{GlcU}(10 \mu \mathrm{M})$ for $15 \mathrm{~min}$, exhibited green fluorescence of the dye produced by GUS-catalyzed hydrolysis in the cytosol. Conversely, no green fluorescence was observed in the absence of dTat-Sar-EED5, GUS or dye-BGlcU. Note that excess GUS and dTat-Sar-EED5 were washed out before dye$\beta \mathrm{GlcU}$ treatment, while excess dye- $\beta \mathrm{GlcU}$ was removed prior to confocal microscopic observation. DIC, differential interference contrast. Scale bars, $40 \mu \mathrm{m}$. 


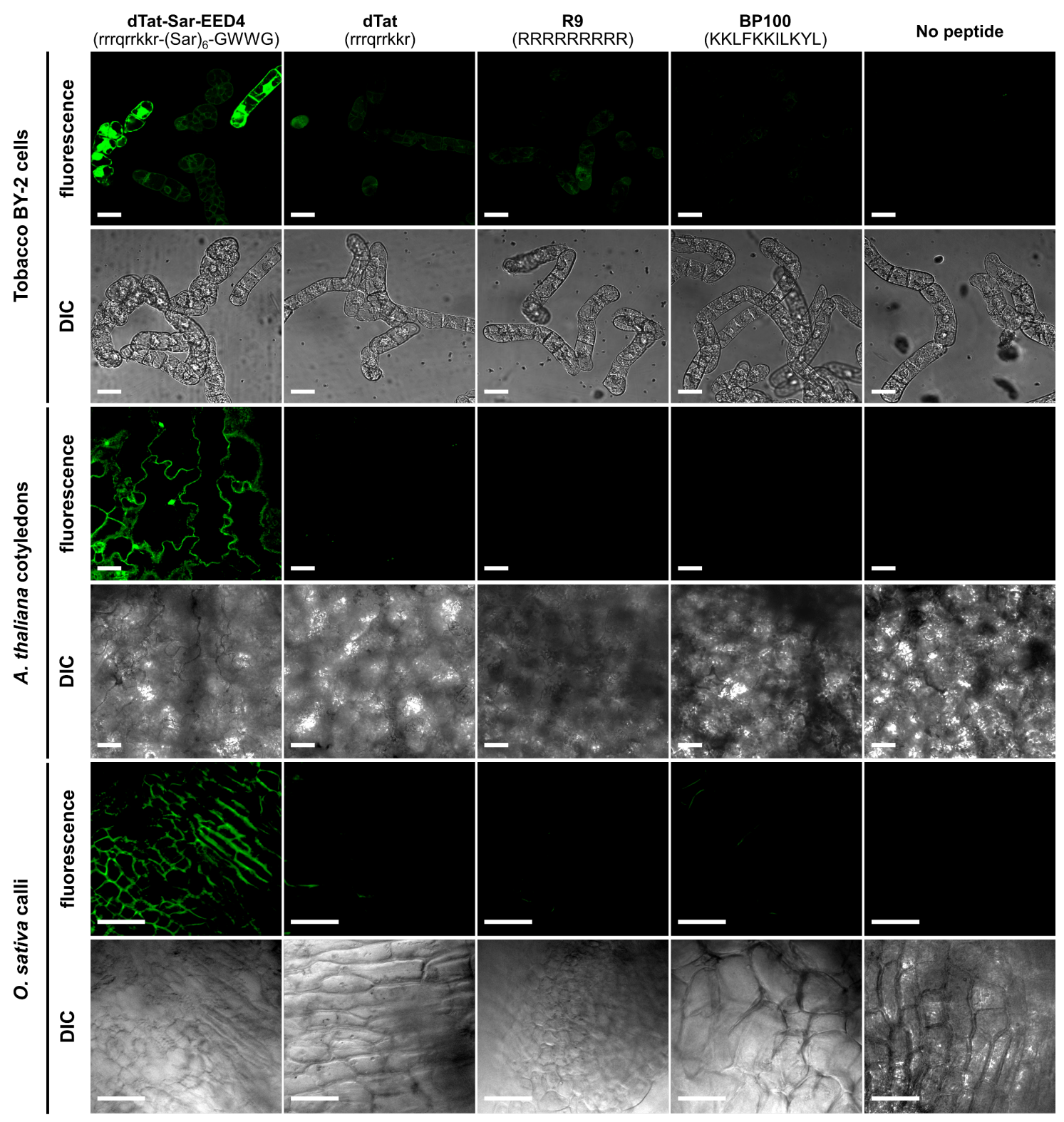

Figure S5. Confocal images of BY-2 cells, $A$. thaliana cotyledons and $O$. sativa calli after GUS transduction with or without various types of peptides (dTat-Sar-EED4, dTat, R9, BP100 or no peptide). Green fluorescence represents the dye produced by GUS-catalyzed hydrolysis in the cells. All fluorescent images are the same as those shown in Fig. $3 b$ in the main text. Differential interference contrast (DIC) images are also shown. Scale bars, $40 \mu \mathrm{m}$. 


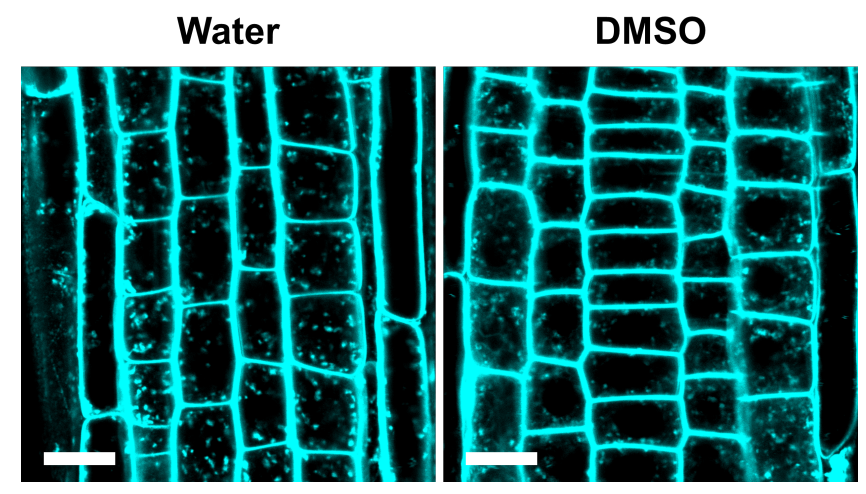

Figure S6. Confocal images of $A$. thaliana root cells treated for 30 min with water or DMSO $(0.6 \% \mathrm{v} / \mathrm{v})$ and then stained for 30 min with FM4-64 $(10 \mu \mathrm{M})$. Scale bars, $50 \mu \mathrm{m}$. The images were obtained from a Leica TCS SP8 confocal microscope (Leica) with Ex/Em wavelengths of 488/620-680 nm. 


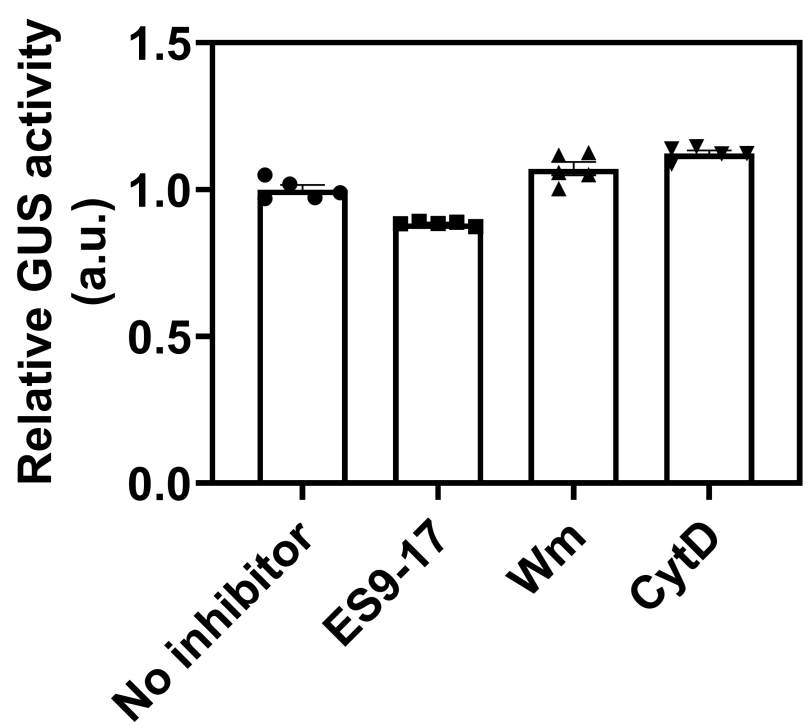

Figure S7. GUS enzymatic activity in the presence of pharmacological inhibitors. The activity under each condition was determined based on the fluorescence intensity of the dye produced by GUS-catalyzed hydrolysis of dye- $\beta \mathrm{GlcU}$ and normalized to that in the presence of inhibitors (No inhibitor). Data from five independent experiments are represented as the mean \pm standard error values. Wm, wortmannin; CytD, cytochalasin D. 


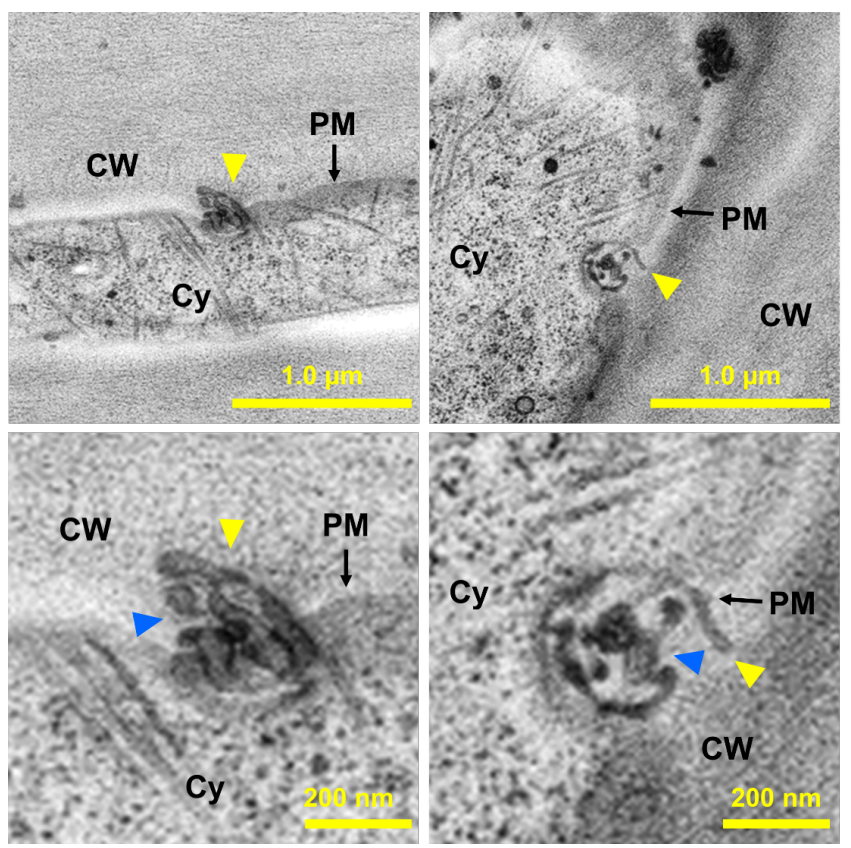

Figure S8. Transmission electron microscopic (TEM) images showing the formation of plasma membrane protrusions with unstructured objects in dTat-Sar-EED4-treated A. thaliana roots. For TEM observation, roots were treated with dTat-Sar-EED4 $(30 \mu \mathrm{M})$ for $15 \mathrm{~min}$ and subjected to high-pressure freezing. Yellow and blue arrowheads indicate membrane protrusions and unstructured objects, respectively. CW, cell wall; $\mathrm{PM}$, plasma membrane; Cy, cytoplasm. 
a
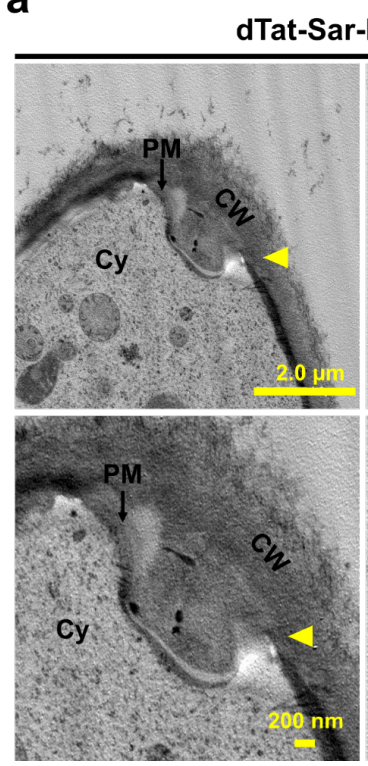

b

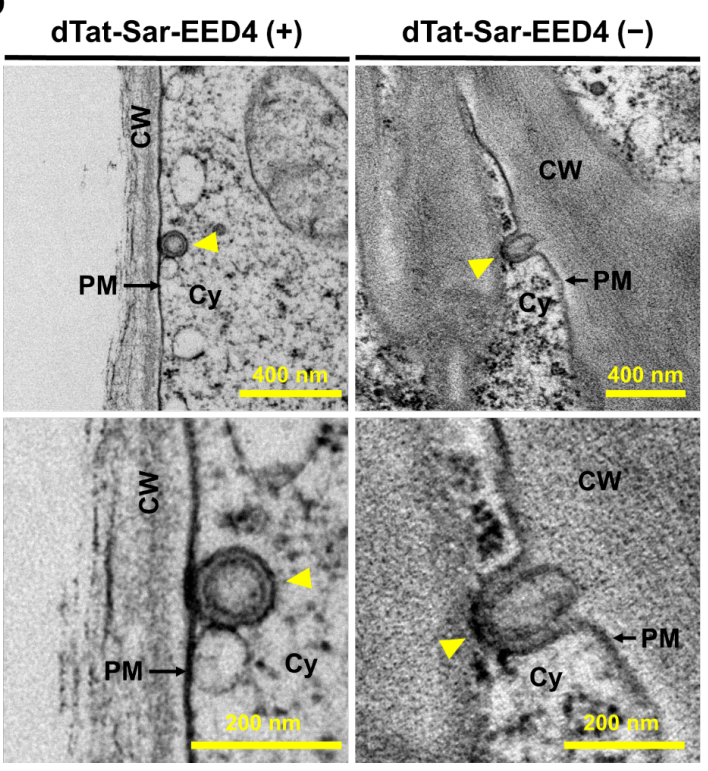

Figure S9. TEM images of BY-2 cells treated without and with dTat-Sar-EED4. a, Formation of plasma membrane protrusions (left) and a large intracellular vesicle (right) observed in TEM images of high-pressure frozen BY-2 cells after treatment with dTat-Sar-EED4 $(30 \mu \mathrm{M})$ for $10 \mathrm{~min}$. Yellow arrowheads indicate plasma membrane protrusions (left) and a large intracellular vesicle ( $>200 \mathrm{~nm}$ in diameter, right). b, Formation of membrane invaginations detected in TEM images of high-pressure frozen BY-2 cells after treatment with dTat-Sar-EED4 $(30 \mu \mathrm{M})$ or water for $10 \mathrm{~min}$. Yellow arrowheads indicate the membrane invagination. $\mathrm{CW}$, cell wall; PM, plasma membrane; Cy, cytoplasm. 


\section{Supplementary Movies}

Movie S1. Citrine uptake by BY-2 cells in the presence of $90 \mu \mathrm{M}$ dTat-Sar-EED4.

Movie S2. Citrine uptake by BY-2 cells in the presence of $90 \mu \mathrm{M}$ dTat as a control. 